\title{
THE
}

\section{A Comparison of Energy Estimators Used in Quantum Monte Carlo Calculations}

Jimmie D. Doll

David L. Freeman

University of Rhode Island, dfreeman@uri.edu

Follow this and additional works at: https://digitalcommons.uri.edu/chm_facpubs

Terms of Use

All rights reserved under copyright.

\section{Citation/Publisher Attribution}

Doll, J. D., \& Freeman, D. L. (1985). A Comparison of Energy Estimators Used in Quantum Monte-Carlo Calculations. J. Chem. Phys., 83(2), 768-771. doi: 10.1063/1.449491

Available at: http://dx.doi.org/10.1063/1.449491 


\title{
A comparison of energy estimators used in quantum Monte Carlo calculations
}

\author{
J. D. Doll \\ Chemistry Division, University of California, Los Alamos National Laboratory, MS G-738, Los Alamos, New \\ Mexico 87545
}

David L. Freeman

Department of Chemistry, University of Rhode Island, Kingston, Rhode Island 02881

(Received 6 December 1984; accepted 14 March 1985)

\begin{abstract}
Path-integral Monte Carlo calculations in quantum statistical mechanics have been performed using either discretized methods or Fourier methods. In each of these methods the internal energy has been calculated using either temperature differentiation or direct operation on the density matrix by the Hamiltonian. It is shown that the variance of the internal energy calculated by operation of the Hamiltonian on the density matrix in the Fourier method is independent of the number of Fourier components included in the expansion of the paths for a number of systems. The variance of the internal energy obtained from the other methods is shown to grow with the size of the expansion used for all systems.
\end{abstract}

\section{INTRODUCTION}

Recently a number of methods have been developed ${ }^{1-8}$ to perform fully quantum mechanical Monte Carlo calculations using the Feynman path integral formulation of statistical mechanics. ${ }^{9}$ The defining expression in these approaches is the path-integral representation of the quantum density operator

$$
\begin{aligned}
& \rho\left(\mathbf{r}_{1}, \ldots, \mathbf{r}_{N} ; \mathbf{r}_{1}^{\prime}, \ldots, r_{N}^{\prime}\right)=\int \mathscr{D} \mathbf{r}_{1}(u) \mathscr{D} \mathbf{r}_{2}(u) \\
& \cdots \mathscr{D} \mathbf{r}_{N}(u) \exp \{-1 / \hbar) \int_{0}^{\beta \hbar} d u\left[1 / 2 \sum_{i=1}^{N} m_{i} \dot{\mathbf{r}}_{i}(u)^{2}\right. \\
& \left.\left.+V\left(\mathbf{r}_{1}(u), \ldots, \mathbf{r}_{N}(u)\right)\right]\right\},
\end{aligned}
$$

from which all equilibrium thermodynamic quantities can be calculated. In Eq. (1) $m_{i}$ is the mass of particle $i$ at coordinate $r_{i}, V$ is the potential energy, $\beta=1 / k_{B} T$, where $k_{B}$ is the Boltzmann constant and $T$ is the temperature, and the path integrals are evaluated over all paths which connect $\mathbf{r}_{1}$ to $\mathbf{r}_{1}^{\prime}$, $\mathbf{r}_{2}$ to $\mathbf{r}_{N}^{\prime}, \ldots$, and $\mathbf{r}_{N}$ to $\mathbf{r}_{2}^{\prime}$. In Monte Carlo studies the path integrals in Eq. (1) are evaluated numerically and two numerical procedures have been proposed. In one method ${ }^{1-5}$ the path integrals are discretized (DISPI) and short interval approximations are introduced. If we let $\mathbf{R}$ be the collective $3 N$-dimensional coordinate for the many-body system in the DISPI method Eq. (1) becomes

$$
\rho\left(\mathbf{R} ; \mathbf{R}^{\prime}\right)=\lim _{P \rightarrow \infty} \rho_{P}\left(\mathbf{R} ; \mathbf{R}^{\prime}\right)
$$

where

$$
\rho_{P}\left(\mathbf{R} ; \mathbf{R}^{\prime}\right)=\int d^{3 N} R_{1} \cdots d^{3 N} R_{P} \prod_{t=1}^{P} \tilde{\rho}\left(\mathbf{R}_{t} ; \mathbf{R}_{t+1}\right)
$$

and

$$
\begin{aligned}
\tilde{\rho}\left(\mathbf{R}_{t} ; \mathbf{R}_{t+1}\right)= & {\left[4 \pi \hbar^{2} \beta / 2 m P\right]^{-3 N / 2} } \\
& \times \exp \left(-P\left(\mathbf{R}_{t}-\mathbf{R}_{t+1}\right)^{2} m / 2 \hbar^{2} \beta\right. \\
& \left.-(\beta / 2 P) V\left(\mathbf{R}_{t}\right)-(\beta / 2 P) V\left(\mathbf{R}_{t+1}\right)\right\} .
\end{aligned}
$$

In Eqs. (2)-(4) $P$ is the number of points used in the discretization of the paths and we take $\mathbf{R}_{1}=\mathbf{R}$ and $\mathbf{R}_{P+1}=\mathbf{R}^{\prime}$. As an alternative to the DISPI method the paths have been expanded in a Fourier series and the integrations over all paths have been replaced by integrations over all Fourier coefficients. ${ }^{6-8}$ In this approach (FOURPI) Eq. (1) becomes

$$
\rho\left(\mathbf{R} ; \mathbf{R}^{\prime}\right)=\lim _{k_{\max } \rightarrow \infty} \rho_{k_{\max }}\left(\mathbf{R} ; \mathbf{R}^{\prime}\right),
$$

where

$$
\begin{aligned}
\rho_{k_{\max }}\left(\mathbf{R} ; \mathbf{R}^{\prime}\right) \\
=J(\beta) \exp \left\{-\left(m / 2 \beta \hbar^{2}\right)\left(\mathbf{R}-\mathbf{R}^{\prime}\right)^{2}\right\} \int_{k=1}^{k_{\max }} d^{3 N} A_{k} \\
\quad \times \exp \left\{-A_{k}^{2} / 2 \sigma_{k}^{2}\right\} \exp \left\{-(1 / \hbar) \int_{0}^{\beta \hbar} V[\mathbf{R}(u)] d u\right\} .
\end{aligned}
$$

In Eq. (6) $\mathbf{A}_{k}$ is the collective $3 N$-dimensional set of Fourier coefficients for component $k$,

$$
\begin{aligned}
& \sigma_{k}=\left[2 \beta \hbar^{2} / m(\pi k)^{2}\right]^{1 / 2}, \\
& J(\beta)=\left(m / 2 \pi \hbar^{2} \beta\right)^{3 N / 2} \prod_{k=1}^{k_{\max }} 1 / \sqrt{2 \pi \sigma_{k}^{2}},
\end{aligned}
$$

and $k_{\max }$ is the index of the maximum Fourier coefficient included. In the FOURPI method the $x$ component of particle $i$ is given by

$$
x_{i}(u)=x_{i}+\left(x_{i}^{\prime}-x_{i}\right) u / \beta \hbar+\sum_{k=1}^{k_{\max }} a_{k x i} \sin (k \pi u / \beta \hbar) .
$$

From the approximate representations of the density matrix several methods have been introduced to calculate the internal energy, $\langle U\rangle$. In one approach ${ }^{7,8,11}(H$ method $)\langle U\rangle$ is calculated as the expectation value of the Hamiltonian

$$
\widehat{H}=-\left(\hbar^{2} / 2 m\right) \sum_{i=1}^{N} \nabla i^{2}+V(\mathbf{R})
$$

over the density matrix to obtain

$$
\langle U\rangle=\int d^{3 N} R\left[\rho\left(\mathbf{R} ; \mathbf{R}^{\prime}\right) \hat{H}\right]_{\mathbf{R}=\mathbf{R}^{\prime}} / \int d^{3 N} R \rho\left(\mathbf{R} ; \mathbf{R}^{\prime}\right) .
$$


In another method ${ }^{1}(T$ method) the energy is obtained from a temperature differentiation to give

$$
\langle U\rangle=-\partial \ln Q / \partial \beta,
$$

where

$$
Q=\int d^{3 N} R \rho(\mathbf{R} ; \mathbf{R})
$$

is the partition function of the system.

The energy obtained from DISPI calculations using the $T$ method has been analyzed by Herman, Bruskin, and Berne $^{4}$ who showed that the variance in the energy obtained in a Monte Carlo evaluation of Eq. (12) grows as the square root of the number of discretization points $P$. In the current note we show that the variance in the energy calculated using the $T$ method in FOURPI calculations grows as the square root of the number of Fourier components included, whereas the variance in the energy using the $H$ method in FOURPI calculations is independent of the number of Fourier coefficients included. In contrast we also show that the variance in the energy using the $H$ method is DISPI calculations grows as the square root of $P$. Consequently only FOURPI calculations using the $H$ method give energies whose variance is independent of the size of the expansion used.

\section{THEORY}

For simplicity we examine the expressions obtained for an ensemble of noninteracting one-dimensional systems. In a DISPI calculation the expression for the energy using the $T$ method is

$$
\begin{aligned}
\langle U\rangle= & -(1 / Q) \int d x d x_{1} \cdots d x_{P} \prod_{t=1}^{P} \tilde{\rho}\left(x_{t} ; x_{t+1}\right)\left\{\sum _ { t = 1 } ^ { P } \left(m P \left(x_{t}\right.\right.\right. \\
& \left.\left.\left.-x_{t+1}\right)^{2} / 2 \hbar^{2} \beta^{2}\right)-(1 / P) \sum_{t=1}^{P} V\left(x_{t}\right)\right\}+P / 2 \beta \quad(14)
\end{aligned}
$$

and the $H$ method gives for the expression for the internal energy $^{10}$

$$
\begin{aligned}
\langle U\rangle= & (1 / Q) \int d x d x_{1} \cdots d x_{P} \prod_{t=1}^{P} \tilde{\rho}\left(x_{t} ; x_{t+1}\right)\{V(x) \\
& -\left(\hbar^{2} / 2 m\right)\left(\left[m P\left(x-x_{1}\right) / \beta \hbar^{2}+(\beta / 2 P) d V / d x\right]^{2}\right. \\
& \left.\left.-(\beta / 2 P) d^{2} V / d x^{2}\right)\right\}+P / 2 \beta
\end{aligned}
$$

In the limit that $P$ becomes infinite Eqs. (14) and (15) become completely equivalent. Consequently from the analysis given by Herman, Bruskin, and Berne $e^{4}$ we know the variance obtained in a Monte Carlo evaluation of both Eqs. (14) and (15) grows as the square root of $P$. As a system becomes increasingly quantum mechanical it is known that an increasing value of $P$ is required to obtain accurate results. From the behavior of the variance the number of Monte Carlo points required also will increase and the computer requirements will grow substantially.

The $T$ method expression for the energy for a one-dimensional system in FOURPI calculations takes the form

$$
\begin{aligned}
\langle U\rangle= & 1 / 2 \beta+\int d x \prod_{k=1}^{k_{\max }} d a_{k} \exp \left\{-a_{k}^{2} / 2 \sigma_{k}^{2}\right\} \\
& \times \exp \left\{-\beta \int_{0}^{1} V[y(u)] d u\right\}\left(k_{\max } / 2 \beta\right. \\
& \left.-\sum_{k=1}^{k_{\max }} a_{k}^{2} / 2 \beta \sigma_{k}^{2}+\int_{0}^{1} V[y(u)] d u\right) \int d x \int_{k=1}^{k_{\max }} d a_{k} \\
& \times \exp \left\{-a_{k}^{2} / 2 \sigma_{k}^{2}\right\} \exp \left\{-\beta \int_{0}^{1} V[y(u)] d u\right\},
\end{aligned}
$$

where

$$
y(u)=x+\sum_{k=1}^{k_{\max }} a_{k} \sin k \pi u .
$$

As Herman, Bruskin, and Berne $e^{4}$ have shown, the growth in the variance in the energy originates in the kinetic energy. The behavior of the variance expected from a Monte Carlo evaluation of Eq. (16) can be observed for the case that $V=0$. Under conditions of zero potential energy the variance in the kinetic energy from Eq. (16) will occur from the Monte Carlo evaluation of

$$
\left\langle\sum_{k=1}^{k_{\max }} a_{k}^{2} / 2 \beta \sigma_{k}^{2}\right\rangle_{k_{\max }}
$$

where the average is evaluated over the distribution given in Eq. (6). If we set

$$
\alpha_{k_{\max }}=\sum_{k=1}^{k_{\max }} a_{k}^{2} / 2 \sigma_{k}^{2} \beta
$$

and define

$$
B(s)=\left\langle\exp \left\{-s \sum_{k=1}^{k_{\max }} a_{k}^{2} / 2 \sigma_{k}^{2}\right\}\right\rangle_{k_{\max }},
$$

then

$$
\left\langle\alpha_{k_{\max }}\right\rangle_{k_{\max }}=-(1 / \beta)(\partial \ln B(s) / \partial s)_{s=0}
$$

and

$$
\begin{aligned}
\left\langle\delta \alpha_{k_{\max }^{2}}^{2}\right\rangle_{k_{\max }} & =\left\langle\alpha_{k_{\max }}^{2}\right\rangle_{k_{\max }}-\left\langle\alpha_{k_{\max }}\right\rangle_{k_{\max }}^{2} \\
& =\left(-1 / \beta^{2}\right)\left(\partial^{2} \ln B(s) / \partial s^{2}\right)_{s=0} .
\end{aligned}
$$

Equation (19) can be evaluated analytically to give

$$
\ln B(s)=-\left(k_{\max } / 2\right) \ln (1+s)
$$

so that

$$
\left\langle\alpha_{k_{\max }}\right\rangle_{k_{\max }}=k_{\max } / 2 \beta
$$

and

$$
\left\langle\delta \alpha_{k_{\max }}^{2}\right\rangle_{k_{\max }}=k_{\max } / 2 \beta .
$$

In analogy with DISPI calculations, $T$ method evaluations of the energy have a variance which grows as the square root of $k_{\max }$, and serious numerical difficulties can be expected as the system becomes increasingly quantum mechanical.

As shown in Ref. 8 the expression for the energy in FOURPI calculations using the $H$ method is 


$$
\begin{aligned}
\langle U\rangle= & 1 / 2 \beta+\int d x \int_{k=1}^{k_{\max }} d a_{k} \exp \left\{-a_{k}^{2} / 2 \sigma_{k}^{2}\right\} \exp \left\{-(1 / \hbar) \int_{0}^{\beta \hbar} V[x(u)] d u\right\}(V(x) \\
& \left.-\left(\hbar^{2} / 2 m\right)\left(\left[(1 / \hbar) \int_{0}^{\beta \hbar}(1-u / \beta \hbar) V^{\prime}[x(u)] d u\right]^{2}-(1 / \hbar) \int_{0}^{\beta \hbar}(1-u / \beta \hbar)^{2} V^{\prime \prime}[x(u)] d u\right)\right) / \int d x \\
& \times \prod_{k=1}^{k_{\max }} d a_{k} \exp \left\{-a_{k}^{2} / 2 \sigma_{k}^{2}\right\} \exp \left\{-(1 / \hbar) \int_{0}^{\beta \hbar} V[x(u)] d u\right\} .
\end{aligned}
$$

For cases where the potential energy vanishes Eq. (26) reduces to the exact result and the variance vanishes for all $k_{\max }$. To observe the behavior of the variance in the energy obtained from Eq. (26) for nonvanishing potentials we consider the case of the harmonic oscillator $\left[V(x)=1 / 2 m \omega^{2} x^{2}\right.$, $\omega=$ the oscillator frequency]. For a harmonic oscillator the $u$ integrations can be evaluated analytically and the variance in the kinetic energy arises from the Monte Carlo evaluation of the expression

$$
\begin{aligned}
I= & \int d x \int_{k=1}^{k_{\max }} d a_{k} \exp \left\{-a_{k}^{2} / 2 \sigma_{k}^{2}-J\left(x, a_{k}\right)\right\} \\
& \times\left(x+2 \sum_{k=1}^{k_{\max }}\left(a_{k} / k \pi\right)\right)^{2} / \int d x \prod_{k=1}^{k_{\max }} d a_{k} \\
& \times \exp \left\{-a_{k}^{2} / 2 \sigma_{k}^{2}-J\left(x, a_{k}\right)\right\},
\end{aligned}
$$

where

$$
\begin{aligned}
J\left(x, a_{k}\right)= & \left(m \omega^{2} \beta / 2\right)\left[x^{2}+1 / 2 \sum_{k=1}^{k_{\max }} a_{k}^{2}\right. \\
& \left.+2 x \sum_{k=1}^{k_{\max }}\left(a_{k} / k \pi\right)\left[1-(-1)^{k}\right]\right] .
\end{aligned}
$$

Equation (27) is in the form of a multivariate Gaussian with a ratio of the variance to the mean of $\sqrt{2}$ independent of $k_{\max }$. To see this explicitly we write Eq. (27) as

$$
\begin{aligned}
I= & \int d y_{0} d y_{1} \cdots d y_{k_{\max }} \exp \left\{-1 / 2 \sum_{k, 1}^{k_{\max }} y_{k} \gamma_{k 1} y_{1}\right\} \\
& \times\left(\sum_{t=0}^{k_{\max }} y_{t} \lambda_{t}\right)^{2} / \int d y_{0} \cdots d y_{k_{\max }} \\
& \times \exp \left\{-1 / 2 \sum_{k, 1}^{k_{\max }} y_{k} \gamma_{k 1} y_{1}\right\},
\end{aligned}
$$

where

$$
\begin{aligned}
& \left(y_{0}, y_{1}, \ldots, y_{k_{\max }}\right)=\left(x, a_{1}, \ldots, a_{k_{\max }}\right), \\
& \gamma_{k k} \begin{cases}m \omega^{2} \beta / 2+1 / \sigma_{k}^{2} & k \neq 0 \\
m \omega \beta / 2 & k=0\end{cases} \\
& \gamma_{0 k}=\left(m \omega^{2} \beta / k \pi\right)\left[1-(-1)^{k}\right], \\
& \gamma_{k 1}=0 \quad \mathrm{k} \text { and } \quad \begin{array}{l}
1 \neq 0 \\
k \neq 1,
\end{array} \\
& \lambda_{0}=1,
\end{aligned}
$$

and

$$
\lambda_{k}=2 / k \pi \quad k \neq 0 .
$$

Equation (29) can be written

$$
\begin{aligned}
I= & \int d p \int d y_{0} \cdots d y_{k_{\max }} \exp \left\{-1 / 2 \sum_{k, 1}^{k_{\max }} y_{k} \gamma_{k 1} y_{1}\right\} p^{2} \delta \\
& \times\left(p-\sum_{t} \lambda_{t} y_{t}\right) / \int d p d y_{0} \cdots d y_{k_{\max }} \\
& \times \exp \left\{-1 / 2 \sum_{k, 1} y_{k} \gamma_{k 1} y_{1}\right\} \delta\left(p-\sum_{t}^{k_{1} 1} \lambda_{t} y_{t}\right) \\
= & \int d p d y_{0} \cdots d y_{k_{\max }} d K \exp \left\{-1 / 2 \sum_{k, 1} y_{k} y_{k 1} y_{1}-i K\right. \\
& \left.\times\left(p-\sum_{i} y_{t} \lambda_{t}\right)\right\} p^{2} / \int d p d y_{0} \cdots d y_{k_{\max }} \\
& \times d K \exp \left\{-1 / 2 \sum_{k, 1} y_{k} \gamma_{k 1} y_{1}-i K\left(p-\sum_{t} y_{t} \lambda_{t}\right)\right\} .
\end{aligned}
$$

We now introduce matrix notation so that Eq. (33) becomes

$$
\begin{aligned}
I= & \int d p d y_{0} \cdots d y_{k_{\max }} d K \exp \left\{-i K p-1 / 2 \mathbf{y}^{T} \gamma \mathbf{y}\right. \\
& \left.+i K \lambda^{T} \mathbf{y}\right\} p^{2} / \int d p d y_{0} \cdots d y_{k_{\max }} \\
& \times d K \exp \left\{-i K p-1 / 2 \mathbf{y}^{T} \gamma \mathbf{y}+i K \lambda^{T} \mathbf{y}\right\} .
\end{aligned}
$$

We now transform the integration variables in Eq. (34) to

$$
\mathbf{y}=\mathbf{b y}^{\prime}
$$

chosen so that

$$
b^{T} \gamma b=1 .
$$

Then Eq. (34) becomes

$$
\begin{aligned}
I= & \int d p d y_{0}^{\prime} \cdots d y_{k_{\max }^{\prime}} d K \exp \left\{-i K p-1 / 2 \sum_{i} y_{i}^{\prime 2}\right. \\
& \left.+i K \sum_{i}\left(\lambda^{T} \mathbf{b}\right)_{i} y_{i}^{\prime}\right\} p^{2} / \int d p d y_{0}^{\prime} \cdots \\
& \times d y_{k_{\max }^{\prime}} d K \exp \left\{-i K p-1 / 2 \sum_{i} y_{i}^{\prime 2}+i K \sum_{i}\left(\lambda^{T} \mathbf{b}\right)_{i} y_{i}^{\prime}\right\} \\
= & \left.\int d p d K p^{2} \exp \left\{-i K p-K^{2}\left(\sum_{i}\left(\lambda^{T} \mathbf{b}\right)_{i}^{2}\right)\right\}\right] \\
& \int d p d K \exp \left\{-i K p-K^{2}\left(\sum_{i}\left(\lambda^{T} \mathbf{b}\right)_{i}^{2}\right) / 2\right\} \\
= & \int d p p^{2} \exp \left\{-p^{2} / 2\left(\sum_{i}\left(\lambda^{T} \mathbf{b}\right)_{i}^{2}\right)\right\} / \\
& \int d p \exp \left\{-p^{2} / 2 \sum_{i}\left(\lambda^{T} \mathbf{b}\right)_{i}^{2}\right\} .
\end{aligned}
$$


Since $I$ is a single Gaussian integral, the ratio of its variance to the mean is $\sqrt{2}$. Consequently the variance of the energy obtained with the $H$ method is FOURPI calculations is independent of $k_{\max }$ for the harmonic oscillator.

\section{CONCLUSIONS}

We have shown that the variance in the energy obtained from Monte Carlo studies using the $T$ method and the $H$ method in DISPI calculations, and using the $T$ method in FOURPI calculations grows with the size of expansion used in the evaluation of the path integrals. In contrast we have shown that the variance of the energy obtained in FOURPI calculations on the harmonic oscillator using the $H$ method is independent of the number $k_{\max }$ of Fourier coefficients included. In our FOURPI studies of the energy of LennardJones systems using the $H$ method, ${ }^{8,11}$ the variance in the energy has always been found to be independent of $k_{\max }$. The variance of the energy in FOURPI calculations using the $H$ method appears to be independent of $k_{\max }$ for a variety of physically important potentials.

An alternative energy estimator has been suggested by use of the virial theorem. ${ }^{4}$ In both FOURPI and DISPI calculations the variance in the energy obtained from the virial theorem is independent of the size of the expansions used in the evaluation of the path integrals. For FOURPI calculations the virial theorem has proved to be deficient in calcula- tions on Lennard-Jones systems. ${ }^{8}$ A similar analysis of the utility of the virial theorem for Lennard-Jones systems in the DISPI method has not yet been given. It is gratifying that energy calculations obtained using the $H$ method in FOURPI studies have proved to be simultaneously useful for hard core systems with a variance that does not grow as the expansion of the paths is increased.

\section{ACKNOWLEDGMENT}

Work at the University of Rhode Island was supported in part by a grant from Research Corporation.

'J. A. Barker, J. Chem. Phys. 70, 2914 (1979).

${ }^{2}$ K. S. Schweizer, R. M. Stratt, D. Chandler, and P. G. Wolynes, J. Chem. Phys. 75, 1347 (1981)

${ }^{3}$ D. Chandler and P. G. Wolynes, J. Chem. Phys. 74, 4078 (1981).

${ }^{4}$ M. F. Herman, E. J. Bruskin, and B. J. Berne, J. Chem. Phys. 78, 5150 (1982).

${ }^{5}$ D. Thirumalai, E. J. Bruskin, and B. J. Berne, J. Chem. Phys. 79, 5063 (1983).

${ }^{6}$ J. D. Doll and L. E. Myers, J. Chem. Phys. 71, 2880 (1979).

7J. D. Doll and D. L. Freeman, J. Chem. Phys. 80, 2239 (1984).

${ }^{8}$ D. L. Freeman and J. D. Doll, J. Chem. Phys. 80, 5709 (1984).

${ }^{9}$ R. P. Feynman, Statistical Mechanics (Benjamin, Reading, Mass., 1972).

${ }^{10}$ E. L. Pollock and D. M. Ceperley, Phys. Rev. B 30, 2555 (1984).

${ }^{11}$ D. L. Freeman and J. D. Doll, J. Chem. Phys. 82, 462 (1985). 
The Journal of Chemical Physics is copyrighted by the American Institute of Physics (AIP). Redistribution of journal material is subject to the AIP online journal license and/or AIP copyright. For more information, see http:/ojps.aip.org/jcpo/jcpcr/jsp Copyright of Journal of Chemical Physics is the property of American Institute of Physics and its content may not be copied or emailed to multiple sites or posted to a listserv without the copyright holder's express written permission. However, users may print, download, or email articles for individual use. 
The Journal of Chemical Physics is copyrighted by the American Institute of Physics (AIP). Redistribution of journal material is subject to the AIP online journal license and/or AIP copyright. For more information, see http://ojps.aip.org/jcpo/jcper/jsp 